Pramāna, Vol. 12, No. 1, January 1979, pp. 9-18, (e printed in India.

\title{
The short range interactions between two rare gas atoms
}

\author{
K L SEBASTIAN* and A K CHANDRA \\ Department of Inorganic and Physical Chemistry, Indian Institute of Science, \\ Bangalore 560012 \\ *Present address: Department of Chemistry, Calicut University, Calicut 673635
}

MS received 27 July 1978

\begin{abstract}
The short range interactions in $\mathrm{He}_{2}, \mathrm{Ne}_{2}$ and $\mathrm{Ar}_{2}$ have been studied in terms of the electronic forces as functions of their internuclear separations employing their single configuration SCF wave functions. The results show that the constituent molecular orbitals behave differently in terms of the forces they exert on the nuclei during the interaction process. The different behaviour of the orbitals is also reflected in the redistribution of charges.
\end{abstract}

Keywords. Electrostatic force; charge equivalent of the electronic force; binding orbitals; atomic overlap; screening terms.

\section{Introduction}

The interactions between two inert gas atoms and between a noble gas atom and its ion have been the object of many theoretical and experimental works. (Amdur et al 1961 ; Ransil 1961 ; Axilrod 1963; Amdur and Jordan 1966; Jordan and Amdur 1967). Besides, an interaction between a noble gas atom and a gas ion is of interest for interpreting phenomena related to self-trapped holes in crystals (Jette et al 1969; Martin 1971). In all such works, the potential energy curves are important. An early single configuration wavefunctions of $\mathrm{He}_{2}$ (Ransil 1961) gave a spurious minimum in the potential energy curve. But Gilbert and Wahl (1967) obtained the single configuration Hartree-Fock (HF) wave functions for $\mathrm{He}_{2}, \mathrm{Ne}_{2}$ and $\mathrm{Ar}_{2}$ by varying the orbital exponents to minimise the energy at each internuclear separation $R$ and did not obtain any minimum in their potential energy curves. However, there is evidence for the formation of weak van der Waals dimers between two inert gas atoms (Tanaka et al 1973). The reason why Gilbert and Wahl (1967) did not obtain the van der Waal's minimum in the potential energy curves of $\mathrm{He}_{2}, \mathrm{Ar}_{2}$ and $\mathrm{Ne}_{2}$ is that the single configuration HF wave functions are not adequate for the proper description of the systems for the extended values of the internuclear distance although they may be quite adequate for the short range of interactions.

In a previous publication, Bader and Chandra (1968) have studied the interaction between two He atoms in terms of the electrostatic force acting on the nuclei employing the single configuration HF wave functions. Earlier, Bader and Preston (1966) have studied the interaction between two He atoms. They correlated the repulsive force to the removal of electronic charge from the internuclear region. They showed that this removal of charge in the three dimensional space is a result of the Pauli exclusion principle, which in fact is an orthogonality restraint in an $n / 2$ or $(n+1) / 2$ 
dimensional space when $\boldsymbol{n}$ is the total number of electrons. Bader and Chandra (1968), using the HF wave functions of $\mathrm{He}_{2}$, have demonstrated this charge removal and attributed this to the operation of the Pauli exclusion principle. The object of this paper is to present results of our similar investigation of the short range interactions in $\mathrm{Ne}_{2}$ and $\mathrm{Ar}_{2}$ as a function of their internuclear separations using the force concept and employing the single configuration HF wave functions. (Gilbert and Wahl 1967). The reason why the force concept is used in such study is that, because of its conceptual simplicity and visuality, it has led to, in the past (Bader and Chandra 1968; Chandra and Sundar 1971; Sundar and Chandra 1974; Chandra and Sebastian 1976a, b), a better understanding of the mechanism of the formation of stable, weakly stable and unstable molecular species from atoms. Besides, the presence of large number of molecular orbitals including the $\pi$-type of orbitals in $\mathrm{Ne}_{2}$ and $\mathrm{Ar}_{2}$ is likely to reveal new features in the interaction pictures. For example, the analysis of the individual molecular orbitals in terms of the electrostatic force should expose the actual role of the inner and outer orbitals in the short range interactions between two heavy inert gas atoms. Furthermore, the formation of $\mathrm{He}_{2}{ }^{+}, \mathrm{Ne}_{2}{ }^{+}$and $\mathrm{Ar}_{2}{ }^{+}$has been established (Conor and Biondi 1965; Aberth and Lorents 1966). Since the formation of these species is a result of short-range interactions the present study is likely to reveal the origin of binding in these molecular systems also.

\section{Interpretation of forces}

A practical method of estimating the net force on a nucleus in a molecule is provided by the Hellmann-Feynman theorem. The net force on a nucleus $A$ in a homonuclear diatomic system separated by the internuclear separation $R$ is given by (Bader and Chandra 1968; Bader and Preston 1966; Chandra and Sundar 1971; Sundar and Chandra 1974)

$$
F_{A}=Z / R^{2}\left(Z-f_{A}^{\rho}\right)
$$

where $Z$ is the charge of each bare nucleus and $f_{A}^{\rho}$ is the charge equivalent of the electronic force acting on a nucleus ${ }^{A}$, and is defined as

$$
f_{A}^{\rho}=R^{2} \int d v \frac{\rho(\bar{r}) \cos \theta_{A}}{r_{A}^{2}}
$$

The electron density $\rho$ at a position $\vec{r}$ can be easily estimated from the wave functions. When a single configuration molecular orbital type of wave functions are used, the total density $\rho$ is given by

$$
\rho=\sum_{i}^{\text {occupied }} n_{i} \phi_{i}^{*}(r) \phi_{l}(r)
$$


where $\phi_{t}$ is a molecular orbital and $n_{i}$ is its occupation number. Hence

$$
f_{A}^{\rho}=\sum_{i}^{\text {occ. }} f_{l_{A}}
$$

where $\quad f_{i A}=R^{2} n_{i} \int \phi_{i}^{*}(r) \phi_{i}(r) d v \frac{\cos \theta_{A}}{r_{A}^{2}}$

For the separated atoms the density distribution corresponding to each nondegenerate molecular orbital (MO) $\phi_{i}$ may be written as

$$
\phi_{i}^{*} \phi_{i}=\frac{1}{2}\left(\phi_{i A}^{2}+\phi_{i B}^{2}\right) \text {, }
$$

where $\phi_{A}$ and $\phi_{B}$ are the pure atomic orbitals on atoms $A$ and $B$ with which $\phi_{i}$ correlates for the separated atoms. Since the centosymmetric orbitals $\phi_{i A}$ cannot contribute to the force on $A$, at the limit of large $R, f_{i A}$ becomes (Sundar and Chandra 1974)

$$
f_{i A}(R \rightarrow \infty)=n_{l} / 2
$$

Therefore the limiting value of $f_{i}$ is 1 for a non-degenerate doubly occupied orbital and 2 for a fully occupied doubly degenerate orbital. The binding, anti-binding and non-binding features of a non-degenerate $M O$ can be characterised by $f_{i}>1, f_{l}<1$ and $f_{i}=1$ respectively. Since for a stable bond $F_{A}=0$ at the equilibrium value of $R$ (i.e. $R e$ ) one may write from (1) and (4) that

$$
f_{A}^{\rho}=\sum_{i}^{\text {occ. }} f_{i A}=Z
$$

For a large diatomic system if the overall interactions lead to chemical binding, some of the orbitals have to be anti-binding in order to achieve the electrostatic equilibrium. Similarly, if the overall interactions lead to repulsion at all values of $R$, some of the orbitals may be binding or non-binding. This is qualitatively expected from the orthogonality restraints of the molecular orbitals. Hence the concept of the colliding orbitals in real space breaks down when dealing with a large number of molecular orbitals in a diatomic system.

It should be mentioned that the evaluation of $f_{i A}$ involves one-centre and two-centre molecular integrals. Methods of evaluation of the two-centre force integrals have been discussed earlier among others by Coulson (1942) and Chandra and Sebastian (1978).

\section{Results and discussion}

3.1. Net force on the nuclei and the electronic forces contributed by the various orbitals

The total forces on each nucleus in $\mathrm{Ne}_{2}$ and $\mathrm{Ar}_{2}$ have been calculated employing the single configuration SCF MO wave functions of Gilbert and Wahl (1967) as they 
seemed to be the best functions available at present. The results are plotted as the functions of $R$ in figure 1. A similar figure for $\mathrm{He}_{2}$ has been published earlier. In figure 1, we have also plotted the derivative of the total energy as a function of $R$. At small values of $R$, the agreement is seen to be poor; however, as $R$ increases the agreement tends to become better. Such a comparison provides a test for the accuracy of wave functions. Since for a given wave function the calculated energy is more accurate than the force obtained as an expectation value of the one-electron operator, the disagreement between the two force-curves in figure 1 arises from the inaccuracy in the wave functions. Since our objective is to understand the nature

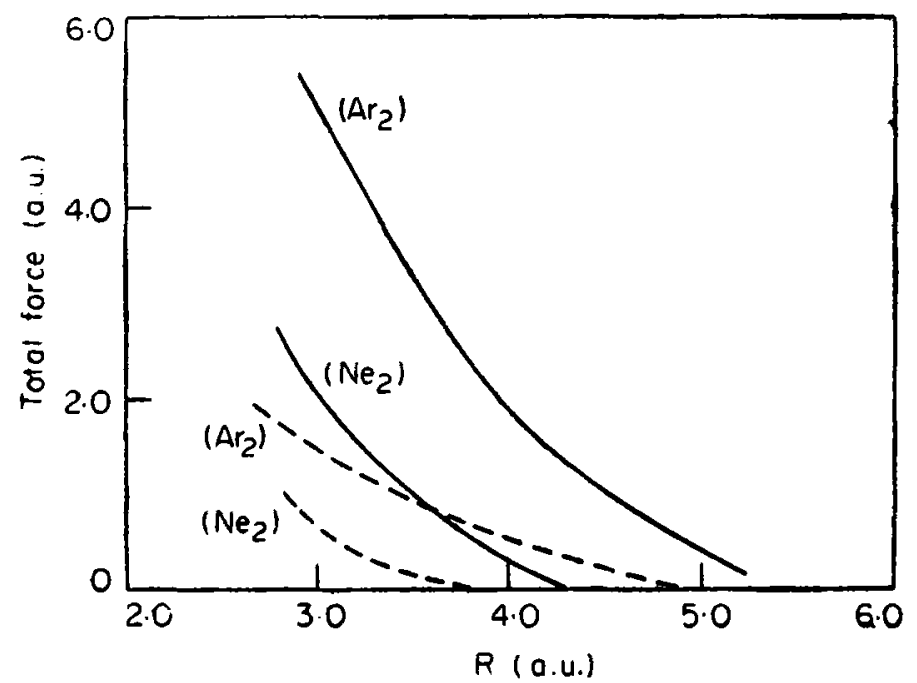

Pigure 1. The plot of total force vs $R$. The dashed lines refer to the derivative of energies.

Table 1. Values of $\left(f_{1}-1\right)$ for the $1 \sigma_{g}$ and $1 \sigma_{u}$ orbitals in $\mathrm{He}_{q}$

\begin{tabular}{ccc}
\hline$R$ a.u. & $1 \sigma g$ & $1 \sigma u$ \\
\hline 1.5 & 0.743 & -1.514 \\
1.75 & 0.783 & -1.423 \\
2.0 & 0.783 & -1.286 \\
2.25 & 0.755 & -1.136 \\
2.50 & 0.706 & -0.984 \\
3.0 & 0.572 & -0.708 \\
\hline
\end{tabular}

Table 2. Values of $\left(f_{t}-1\right)$ for the $\sigma *$ and $\left(f_{t}-2\right)$ for the $\pi$ - MOs in $\mathrm{Ne}_{2}$

\begin{tabular}{ccccccccc}
\hline$R$ a.u. & $1 \sigma g$ & $1 \sigma u$ & $2 \sigma g$ & $2 \sigma u$ & $3 \sigma g$ & $1 \pi u$ & $1 \pi g$ & $3 \sigma u$ \\
\hline 2.5 & -0.055 & -0.061 & -0.041 & -0.025 & 1.836 & 0.114 & -0.618 & -3.508 \\
2.8 & -0.048 & -0.055 & --0.072 & -0.025 & 1.809 & 0.095 & -0.488 & -2.783 \\
$\mathbf{3 . 0}$ & -0.039 & -0.048 & -0.074 & -0.018 & 1.731 & 0.079 & -0.417 & -2.383 \\
3.5 & -0.021 & -0.035 & -0.060 & 0.006 & 1.320 & 0.048 & -0.281 & -1.602 \\
4.0 & $\mathbf{0 . 0 0 0}$ & -0.019 & -0.047 & 0.021 & 1.067 & 0.006 & -0.191 & -1.054 \\
$\mathbf{5 . 0}$ & $\mathbf{0 . 0 0 0}$ & -0.009 & -0.024 & 0.023 & 0.551 & -0.026 & -0.093 & -0.416 \\
\hline
\end{tabular}




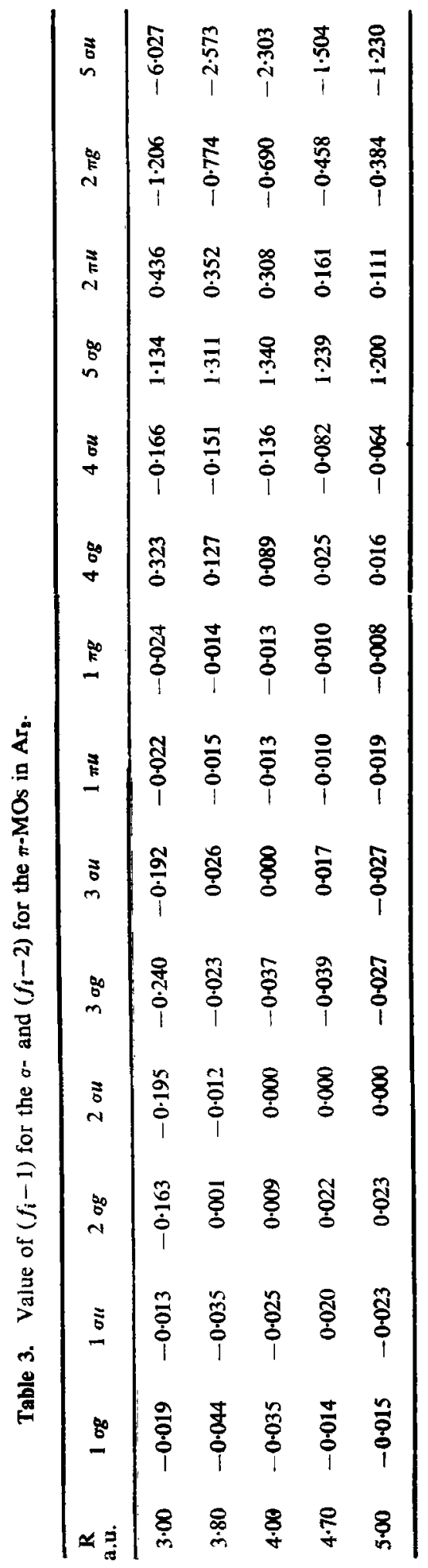


of interactions between two atoms as a function of $R$, such an inaccuracy will not seriously affect the qualitative nature of our conclusions.

The results of figure 1 show that the interactions between two inert gas atoms are always repulsive. But the behaviour of the component MOs can be understood from the relative magnitude of $\left(f_{t}-1\right)$ for the $\sigma$-orbitals and $\left(f_{i}-2\right)$ for the $\pi$-orbitals. They are given for $\mathrm{He}_{2}, \mathrm{Ne}_{2}$ and $\mathrm{Ar}_{2}$ in tables 1, 2 and 3 respectively where the MOs are arranged in the order of increasing energy.

The results for $\mathrm{He}_{2}$ are very straightforward and in conformity with the traditional concept that the $\sigma g$ and $\sigma u$ orbitals are respectively binding and anti-binding. Since the anti-binding force of the $1 \sigma u$ orbital dominates the binding force of the $1 \sigma \mathrm{g}$ orbital for any given value of $R, \mathrm{He}_{2}$ with the electron configuration $1 \sigma g^{2} 1 \sigma u^{2}$ is always unstable. The results of tables 2 and 3 however show that, contrary to the traditional concept, the inner orbitals in $\mathrm{Ne}_{2}$ and $\mathrm{Ar}_{2}$ are slightly anti-binding regardless of their $\sigma g$ and $\sigma u$ symmetry. Such orbitals which form essentially a core round the nuclei are polarised behind the nuclei. This back-polarisation is responsible for the anti-binding character of the inner orbitals and is also noted for the core electrons in $\mathrm{Li}_{2}$ (Sundar and Chandra 1974). The outer orbitals are however binding and anti-binding depending on their symmetries. For example, in $\mathrm{Ne}_{2}$, the $3 \sigma g$ and $1 \pi u$ orbitals are binding while the $3 \sigma \mathrm{u}$ and $1 \pi g$ orbitals are anti-binding. It could be easily shown from tables 2 and 3 that the binding or anti-binding force for each $\pi$ electron is less than that for each $\sigma$-electron. This is expected because the $\pi$-electron clouds are above and below the internuclear axis with a node on it while the $\sigma$-electron clouds are mostly localised along the internuclear axis.

\subsection{The partition of forces and their interpretation}

In order to understand the roles of the outer orbitals in the interactions process, $f_{i}$ for each orbital is split into three components as

$$
f_{i A}=f_{i A}^{A A}+f_{i A}^{A B}+f_{i A}^{B B}
$$

where these three components are respectively called the atomic, overlap and screening terms. The physical significance of each term in (9) has been discussed earlier (Bader and Chandra 1968; Sundar and Chandra 1974). The values of each term for $3 \sigma g 3 \sigma u, 1 \pi u$ and $1 \pi g$ in $\mathrm{Ne}_{2}$ and their variations with $R$ are shown in figure 2 . It is seen that $3 \sigma \mathrm{g}$ is polarised inwards causing an attractive force on the nucleus. The overlap term is large and positive suggesting the transfer of density in the internuclear region. The variation of the screening term shows a very small amount of descreening of the nuclear charge by the $3 \sigma g$ density at $R=2.5$ a.u. Therefore, the binding nature of the $3 \sigma g \mathrm{MO}$ is primarily due to the transfer of density in the internuclear region. On the other hand, the $3 \sigma u \mathrm{MO}$ is polarised behind the nuclei and almost cancels the small positive atomic force contributed by the $3 \sigma g$ density. The screening term of $3 \sigma u$ is greater than the limiting value of unity at small $R$. Owing to the presence of a node in the $3 \sigma u \mathrm{MO}$, a nucleus cannot penetrate into the charge cloud of the other (Chandra and Sebastian 1978). Hence, the nuclear charge cannot be descreened and the increase of the screening term over the limiting value at small $R$ is due to the possible contraction of atomic densities near the nuclei. 


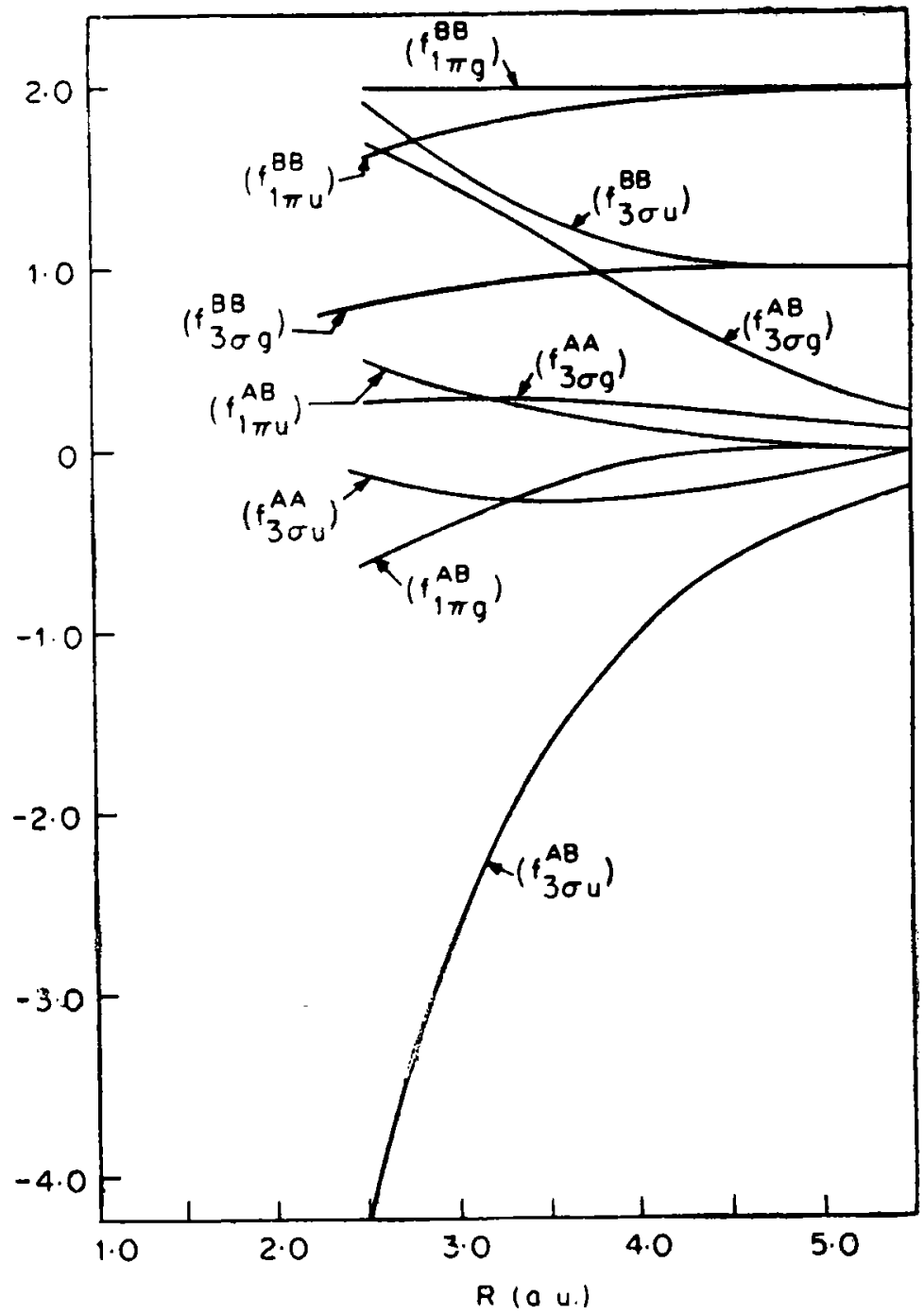

Figure 2. The variations with $R$ of the atomic, overlap, and screening terms for some $\mathrm{MOs}$ in $\mathrm{Ne}$.

The large negative overlap term implies that the charge density is transferred behind the nuclei, resulting in a force that pulls the nuclei away from each other. Since electrons in the inner orbitals screen the nuclear charge quite effectively a $3 \sigma u$ electron in $\mathrm{Ne}_{2}$ will experience an effective nuclear charge of +1 . But the value of $f_{3 \sigma u}^{A B}$ at $R=2 \cdot 5$ au implies that the $3 \sigma u$ density pulls the nuclei away from one another with a force four times greater than the force of repulsion between two nuclei of charge +1 separated by the same value of $R$. This is a very large effect and makes the $3 \sigma u$ MO highly anti-binding.

The behaviour of the $\pi$-MOs are quite different. Since the $\pi$-orbitals are perpendicular to the internuclear axis there will be no polarisation in the direction of the bond. In $1 \pi u$, the nuclear charge is descreened as $R$ is decreased and the resulting 
force of repulsion is almost balanced by the migration density to the internuclear region. So the $1 \pi u \mathrm{MO}$ is almost non-binding. In $1 \pi g$, the screening term is always approximately, 2 which is the limiting value for the doubly degenerate MO. The negative overlap term whose magnitude increases as $R$ is decreased, indicates that electrons migrate behind the nuclei causing the repulsion.

\subsection{Formation of molecular ions}

The presence of the binding and anti-binding orbitals in $\mathrm{He}_{2}, \mathrm{Ne}_{2}$ and $\mathrm{Ar}_{2}$ suggest that if an electron with a very high anti-binding ability is removed the binding may result and the stable molecular ions $\mathrm{He}_{2}{ }^{+}, \mathrm{Ne}_{2}{ }^{+}$and $\mathrm{Ar}_{2}{ }^{+}$could be formed. It is seen from the tables that in all the cases the highest occupied orbital is most anti-binding. If, an electron from such orbital is removed, the binding may result. In figure 3 is plotted the quantity

$$
\sum_{i}^{\text {occ. }}\left(f_{i A}-Z\right) \text { vs } R
$$

for the wave functions of $\mathrm{He}_{2}, \mathrm{Ne}_{2}$ and $\mathrm{Ar}_{2}$ where the prime in the summation refers to the removal of an electron from the highest occupied orbital. For the stable molecular ion

$$
\sum_{i}^{\infty c c .}\left(f_{t A}-Z\right)=0
$$

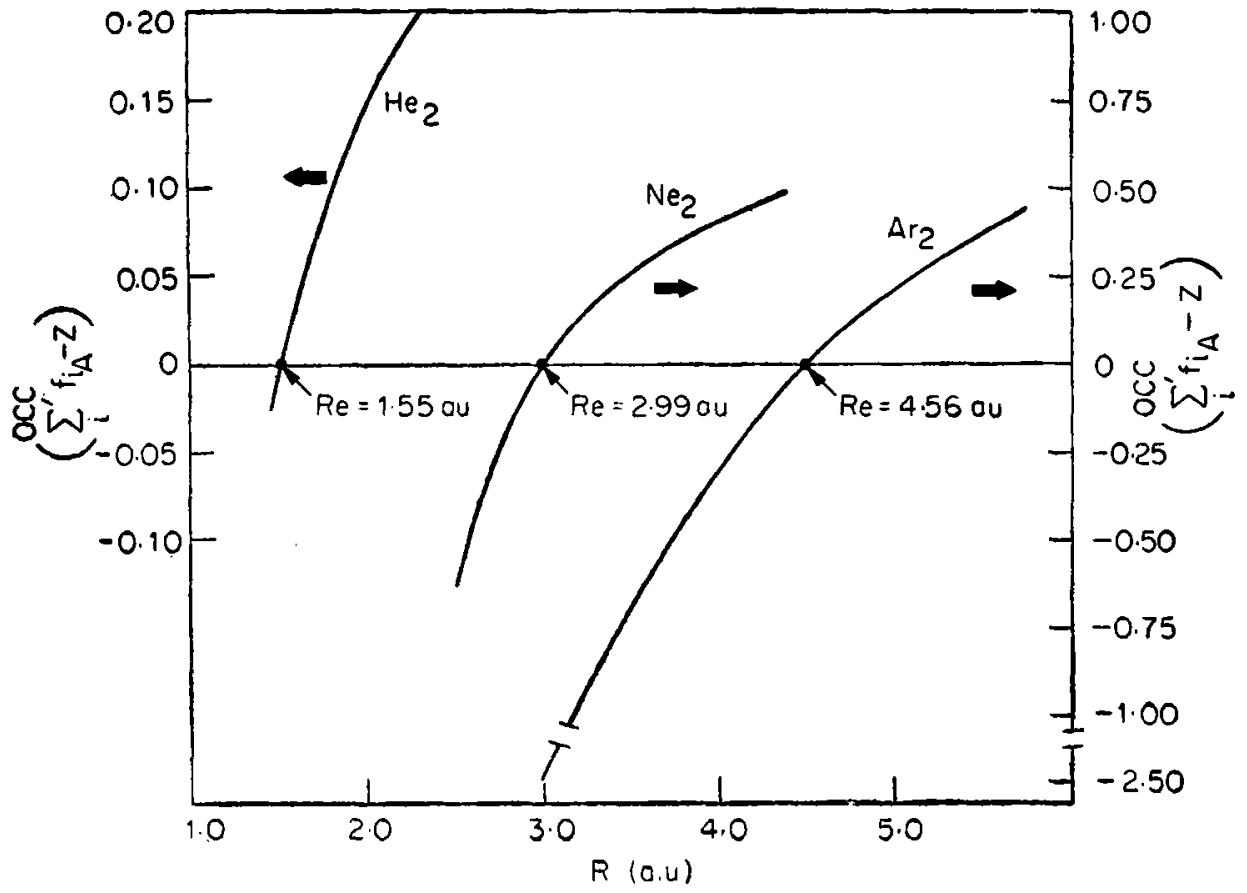

Figure 3. The plot for the wave functions of $\mathrm{He}_{2}, \mathrm{Ne}_{2}$ and $\mathrm{Ar}_{2}$. 
and the equilibrium value of $R$ for the molecular ions is shown in figure 3 . It is very striking that these anticipated values of $\mathrm{Re}$, especially for $\mathrm{Ne}_{2}{ }^{+}$and $\mathrm{Ar}_{2}{ }^{+}$, are quite close to those obtained by Gilbert and Wahl (1971) from the single configuration $\mathrm{HF}$ wave functions of the ${ }^{2} \Sigma_{u}$ state of $\mathrm{He}_{2}{ }^{+}\left(R_{e}=2.0 \mathrm{au}\right) \mathrm{Ne}_{2}{ }^{+}\left(R_{e}=3.2 \mathrm{au}\right)$ and $\operatorname{Ar}_{2}{ }^{+}\left(R_{e}=4.6 \mathrm{au}\right)$ where the orbital exponents are optimised for each value of $R$. This demonstrates that for many electron systems the wave functions are not altered much when an electron is removed from the highest occupied orbital.

\subsection{The density difference diagrams}

Figures 4 and 5 give the density difference, i.e., $\triangle \rho$ contour maps for $\mathrm{Ne}_{2}$ and $\mathrm{Ar}_{2}$ respectively. The $\triangle \rho$-contour maps for $\mathrm{He}_{2}$ (Bader and Chandra 1968) are published earlier. It is noted that these diagrams for $\mathrm{Ne}_{2}$ and $\mathrm{Ar}_{2}$ are more complicated than those for $\mathrm{He}_{2}$. As in the case of $\mathrm{He}_{2}$, both these systems are however characterised by a removal of charge from the internuclear region. $\mathrm{He}_{2}$ has only one region of density decrease i.e., the internuclear region. But in $\mathrm{Ne}_{2}$ and $\mathrm{Ar}_{2}$, the density decrease occurs in more than one region. For example, figure 4 for $\mathrm{Ne}_{2}$ shows that the density-decrease occurs not only from the central part of the internuclear region but also from a region close to each nucleus. This map also shows that there is a region immediately in front of the nuclei where the density build-up takes place. This reflects the presence of a strong binding orbital among the various MOs of $\mathrm{Ne}_{2}$.

Figure 5 shows the $\triangle \rho$-map for $\mathrm{Ar}_{2}$ which is still more complicated than figure 4 owing to the presence of larger number of the mutually orthogonal MOs than in
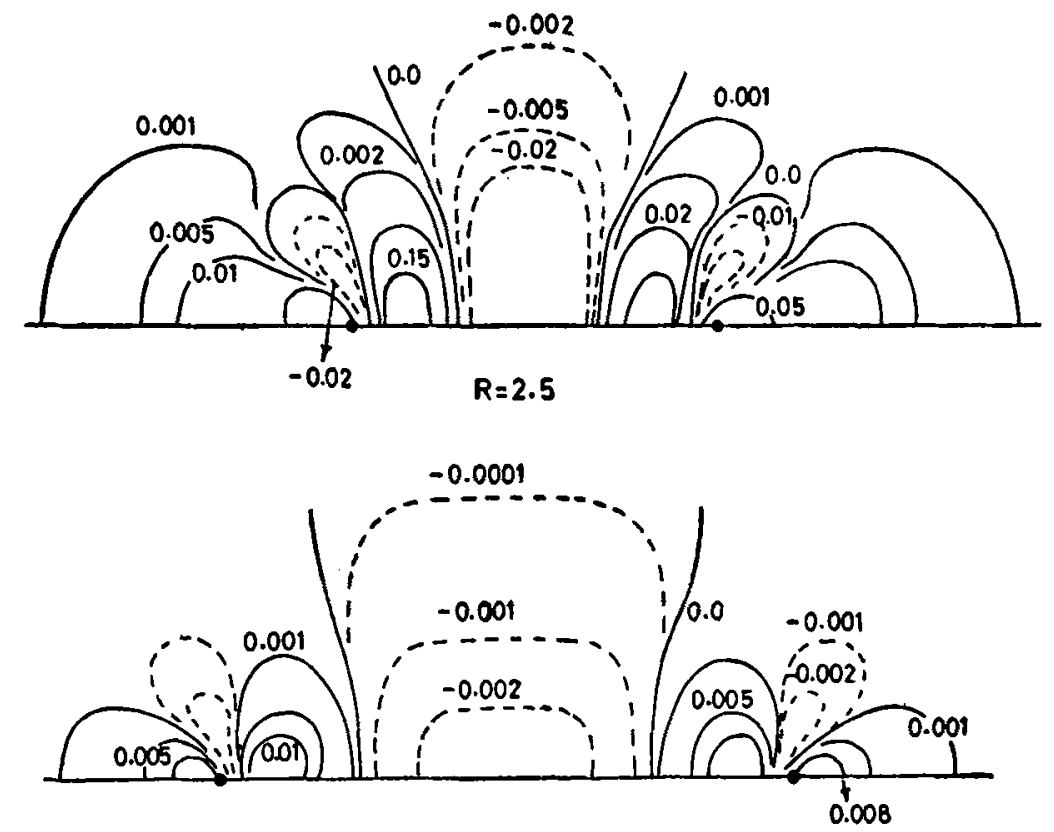

$R=4.0$

Figure 4. The $\Delta \rho$-map for $\mathrm{Ne}_{2}$ at the indicated values of $R$, 


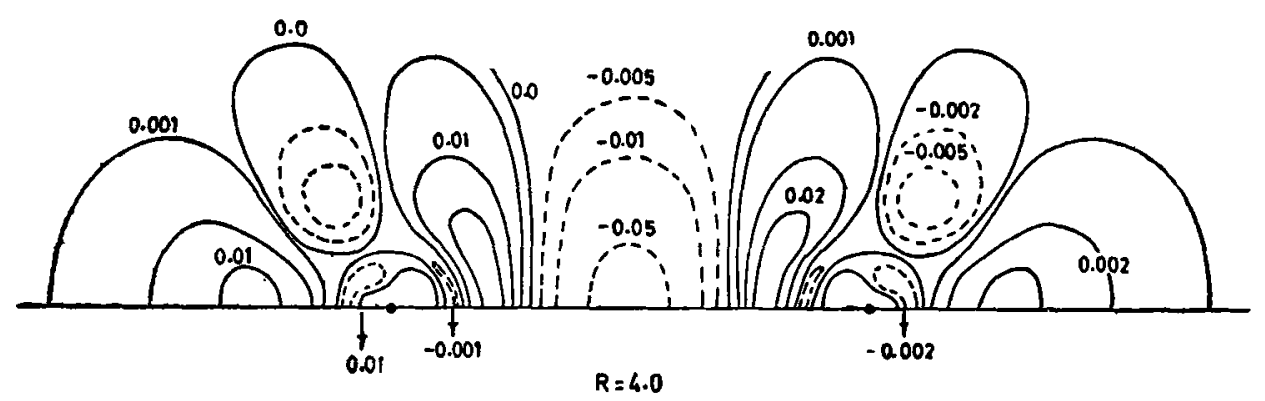

Figure 5. The $\Delta \rho$-map for $\mathrm{Ar}_{\mathrm{a}}$ for $R=4 \cdot 0$ au.

$\mathrm{Ne}_{2}$. Figure 5 shows $\mathrm{Ar}_{2}$ has more regions of the density-decrease than $\mathrm{Ne}_{2}$. In general, the results show that the binding, non-binding and anti-binding nature of the various orbitals are reflected in the redistribution of charges during the interaction process.

\section{Conclusions}

The interaction between two rare gas atoms is repulsive. The repulsion arises primarily from the density of the highest occupied $\sigma$-orbital which exerts a strong anti-binding force on each nucleus. The inner orbitals are slightly anti-binding at small values of the internuclear separations. The presence of a strong binding orbital in the outer shell of the systems leads to the formation of stable molecular ions when an electron is removed from the highest occupied orbital.

\section{References}

Amdur I, Jordan J E and Colgate S O 1961 J. Chem. Phys. 341525

Amdur I and Jordan J E 1966 Adv. Chem. Phys. 1029

Aberth W and Lorents D C 1966 Phys. Rev. 144109

Axilrod B M 1963 A survey of some empirical and semi-empirical interatomic and intermolecular potentials (Washington: Natl. Bur. Stds)

Bader R F W and Chandra A K 1968 Can. J. Chem. 46953

Bader R F W and Jones G 1961 Can. J. Chem. 391253

Bader R F W and Preston H J T 1966 Can. J. Chem. 431131

Chandra A K and Sebastian K L 1976a Mol. Phys. 311489

Chandra A K and Sebastian K L 1976b Chem. Phys. Lett. 41593

Chandra A K and Sebastian K L $1978 \mathrm{Mol}$. Phys. (in press)

Chandra A K and Sundar R 1971 Mol. Phys. 22369

Connor R and Biondi M A 1965 Phvs. Rev. 140A 778

Coulson C A 1942 Proc. Cambridge Philos. Soc. 38210

Gilbert T L and Wahl A C 1967 J. Chem. Phys. 473425

Gilbert T L and Wahl A C 1971 J. Chem. Phys. 555247

Jette A N, Gilbert T L and Das T P 1969 Phys. Rev. 184884

Jordan J E and Amdur I 1967 J. Chem. Phys. 46165

Martin M 1971 J. Chem. Phys. 543289

Ransil B J 1961 J. Chem. Phys. 342109

Sundar R and Chandra A K 1974 Indian J, Chem. 12145

Tanaka S, Yoshino K and Freeman D E 1973 J. Chem. Phys. 59564 